\title{
Sickle Cell Anemia: A Succinct Look into Recently Tested Disease Modifying Interventions
}

\author{
Malena Allbright ${ }^{1}$, Antonio Fernandez ${ }^{1 *}$ \\ ${ }^{1}$ College of Nursing and Health Sciences, Barry University, Miami Shores, FL, USA. \\ *Corresponding Author: Antonio Fernandez, College of Nursing and Health Sciences, Barry University, \\ Miami Shores, FL, USA, Tel: +305-899-3846; Email: anfernandez@barry.edu
}

Received Date: 14-04-2020; Accepted Date: 08-05-2020; Published Date: 15-05-2020

Copyright $^{\circledR} 2020$ by Fernandez A, et al. All rights reserved under CC BY-NC-ND. This is an open access article distributed under the terms of the Creative Commons Attribution License, which provides freedom to read, share, copy and redistribution of material in any of the medium, provided with the original author and source are credited.

\begin{abstract}
Sickle Cell Anemia (SCA) is a globally prevalent, monogenic, life-threatening blood disorder with a complex pathology that remains obscure. A deepened understanding of the malady in the recent years has led to pharmaceutical advancements that target pathophysiology and ultimately ameliorate associated multivariate clinical manifestations. Abnormal cell to cell interactions, endothelial adhesion, induced oxidative stress, intracellular erythrocyte dehydration and concentration levels of fetal hemoglobin are a few factors know to play a key role in microvascular obstruction in individuals with hemoglobin S. All of which have recently been manipulated in-vitro to pharmaceutically alter clinical complications, disease progression and mortality patterns.

The purpose of this paper is to provide a condensed overview of recent discoveries made within SCA pathology and subsequent pathologic based therapeutic agents. A general overview of the molecular basis of the malady, global prevalence and the efforts toward advancing stem cell transplantation technology will also be discussed.
\end{abstract}

\section{Keywords}

Sickle Cell Disease; Hemoglobin; Hydroxyurea; Allogenic Hematopoietic Stem Cell 


\section{Introduction}

Sickle Cell Disease (SCD) denotes a group of pleiotropic hemoglobinopathies that mechanically affect the body's vasculature. Of the various forms, Sickle Cell Anemia (SCA) or homozygous Hemoglobin $\mathrm{S}(\mathrm{HbS})$ is the most common. It is characterized by the production of abnormally shaped red blood cells, which can obstruct and damage circulation, initiating a cascade of atypical cell interactions [1]. Decreased organ perfusion to the kidneys, spleen, nervous, cardiovascular, respiratory and urinary systems become imminent in the absence of appropriate pathophysiology specific therapy [2]. Sickle cell crisis is the most prevalent clinical complication associated with SCA and usually precedes multivariate symptoms correlating with decreased organ perfusion and functioning.

There are 100 million carriers of heterozygous Hemoglobin AS or Sickle Cell Trait (SCT) worldwide. Global prevalence suggests a long standing selective pressure against falciparum malaria and consecutive carrier migration from malaria endemic regions [3]. SCT carriers demonstrate minimal, if any clinical complications throughout one's lifetime. Nevertheless, 300,000 babies are born to these carriers each year with a $25 \%$ chance of their children inheriting SCD. The benefits of SCT genotyping and fetal screening has directly impacted mortality and morbidity patterns in resource accessible regions [4]. Limited access to frontline therapies and proper medical follow in malaria endemic and developing regions are great attributors to disease prevalence. Moreover, a deepened understanding of the complex pathology of SCA in the past decade has encouraged researchers to shy away from panoptic therapies and gear toward development of pathology- modifying interventions that ultimately decline global incidences on a greater scale.

Inheritance of the sickle cell gene causes a non-conservative missense mutation at the beta globin chain [5]. The exchange of hydrophobic valine with hydrophilic glutamic acid on the $6^{\text {th }}$ position of beta globin induces hemoglobin $S$ to sickle in anoxic and acidic microenvironments; also referred to as the deoxy-sickle hemoglobin phase. Mechanical obstruction of the micro-vessels by recurring red cell sickling has been typically estimated as the sole reason for decreased organ perfusion and tissue infarction; yet recent scientific progress proves otherwise. Further demystification of the mechanistic complexity of $\mathrm{HbS}$ has led to pharmaceutical advancements aimed to target abnormal endothelial interactions, inflammation and red cell dehydration in individuals with the life compromising disease. Manipulation of vasoactive factors in-vitro has demonstrated encouraging results for new oral therapeutic agents anticipated to impact individuals in developing regions [6,7].

Frontline SCD therapies such as hydroxyurea and blood transfusions vary in clinical efficacy from patient to patient and is not readily accessible in impoverished regions. Stem cell transplantation is the only curable option for SCD yet advancements in donor transplantation and remain limited. Although a thorough investigation of the complex, multivariate pathophysiology of SCD does not fall within the scope of this review, emphasis will be placed

Fernandez A | Volume 1; Issue 2 (2020) | JCMR-1(2)-010 | Review Article

Citation: Fernandez A, et al. Sickle Cell Anemia: A Succinct Look into Recently Tested Disease Modifying Interventions. Jour Clin Med Res. 2020;1(2):1-10. 
on disease targeting agents that are being studied to date that perpetuate a less hindering clinical outcome in those suffering from SCA.

\section{Increasing Fetal Haemoglobin Concentration}

Increased levels of fetal hemoglobin $(\mathrm{HbF})$ has long been understood to improve SCA related clinical complications. Having 2 alpha and 2 gamma chain tetramers physiologically inhibits $\mathrm{HbF}$ from entering the deoxy-sickle hemoglobin phase. Contrary to adult hemoglobin, fetal hemoglobin decreases the likelihood of $\mathrm{HbS}$ polymerization [8]. During gestation, fetal hemoglobin predominates until its regression to less than $1 \%$ at nearly 6 to 12 months after birth. This phenomenon accounts for why most SCD infants are asymptomatic in the first few months [9]. Moreover, baseline fetal hemoglobin concentrations fluctuate amongst haplotypes of the sickle cell gene. Senegal and Saudi-Indian haplotypes manifest higher HbF levels in adulthood and thus demonstrate a milder clinical expression contrary to the other beta S-globin haplotypes [10]. Pharmacologically inducing fetal hemoglobin levels strongly correlates with a reduction in episodes of pain crises, hemolysis and longer survival [11].

For over 30 years, Hydroxyurea (HU) remains the therapeutic standard for modifying SCD pathogenesis and increasing $\mathrm{HbF}$. $\mathrm{HU}$ is mechanistically known to inhibit the formation of deoxyribonucleotides, thereby trapping cells in the synthesis stage of the cell cycle and stimulating erythropoiesis [12]. Consequentially, the intercalation of fetal and adult hemoglobin $(\mathrm{HbA})$ hinders the polymerization of $\mathrm{HbS}$ and subsequent red cell sickling. $\mathrm{HU}$ related studies have also proved that clinical efficacy is not only a result of $\mathrm{HbF}$ production but also a demotion of abnormal blood cell interactions, endothelial adherence, blood viscosity and premature intravascular hemolysis [13]. The Multi-Center Study of Hydroxyurea (MSH) was a prominent placebo controlled randomized trial for adults in 1999 that revealed a $44 \%$ reduction in pain crisis in the placebo group. Furthermore, fewer episodes of acute chest syndrome, splenic sequestration, transfusion induced hospitalizations and priapism were also reported [14]. In 2011, HU was considered by the Food and Drug Administration (FDA) to be an appropriate therapy for children over 2 years old. The BABY HUG study was the first placebo-controlled randomized trial of $\mathrm{HU}$ in adolescents that even included infants with mild clinical complications. Of note, the placebo group demonstrated a 5 times higher dactylitis occurrence and 3 times higher incidence of acute chest syndrome [15].

Hydroxyurea for children is arguably underused in malarial-endemic regions where the greatest sickle cell burden prevails [16]. The NOHARM, placebo controlled multicenter study was one of the first of its kind to test the efficacy of HU on children in malaria-endemic Uganda. HU recipients had demonstrated benefits similar to trials performed in non-malarious regions; including a less frequent SCA-related clinical outcome, a notable decrease in reticulocyte formation, splenic sequestration and the need for blood transfusion compared to the placebo group [17]. Despite the encouraging results, the demand for optimal HU dosing and proper regimen monitoring remains a challenge. With the maximum dosage being $35 \mathrm{mg} / \mathrm{kg}$ in order to avoid cytopenia and toxicity, $\mathrm{HbF}$ induction via hydroxyurea is considered lower than the Fernandez A | Volume 1; Issue 2 (2020) | JCMR-1(2)-010 | Review Article 
magnitude expected to completely prevent SCA related clinical complications [18]. In fact, studies have proven that $\mathrm{HbF}$ induction via Hydroxyurea varies amongst individuals with reports of baseline regression status post 2 years of treatment [19]. Moreover, rates of nonadherence to HU secondary to an unvaried treatment outcome is also a contributor of the delayed decline in disease prevalence [20]. Thus, the need for a robust HbF inducing agent is imminent in effectively treating mortality rates in heath-impoverished regions.

Pharmacological advancements in HbF biochemistry resulted in the genetic manipulation of HbF gene silencer, DNMT1. 5-aza-2'-deoxycytidine (decitabine) functions to bind and deplete DNMT1 and therefore upregulate fetal hemoglobin in erythrocytes [21]. The first human study on the efficacy of decitabine in HbF induction was performed in 2017 and demonstrated an encouraging outcome for larger trials forthcoming. The randomized trial administered decitabine with the oral multidrug resistant transporter, tetrahydrouridine (THU) in order to expand bioavailability and counteract pharmacologic limitations. The randomized, phase I/II study revealed an increase in fetal hemoglobin levels to $80 \%$ of total erythrocytes in the treated group. A decline in levels of cellular adhesion and reticulocyte production were also noted [22]. Although decitabine has potential toxicities as does $\mathrm{HU}$, this $\mathrm{HbF}$ inducer demonstrates a greater efficacy even in patients non-responsive to Hydroxyurea. While the demand for more research remains, oral interventions that genetically manipulate the $\mathrm{HbF}$ gene may allow less follow-up need in regions that may not have access.

\section{Oxidative Stress and Vascular Tone}

The aberrant biochemistry of SCA promotes premature intravascular hemolysis. This occurs when sickled red cells release free hemoglobin polymers into the blood plasma via the free hemoglobin scavenging mechanism. This consequentially impairs the synthesis and circulation of nitric oxide thus impedes on vascular constriction [23]. Normal levels of nitric oxide maintain vascular tone by stimulating the vascular endothelium to relax. Accumulation of hemoglobin in the plasma are reported to be avid scavengers of nitric oxide thus reducing its bioavailability [24]. Moreover, nitric oxide depletion is a precursor to vasospasms even in larger vessels. Inability to maintain a proper vascular tone attributes to SCA-related cerebrovascular disease, priapism and chronic leg ulcers [25]. Progressive elevation of pulmonary vascular tone and vascular remodeling are also commonly manifested in individuals with SCA. In one study, 20 autopsies were performed on individuals with Hemoglobin S. It was reported that $75 \%$ of these individuals demonstrated signs of pulmonary hypertension [26]. Pharmaceutical prevention of intravascular hemolysis directly correlates with ameliorating clinically related outcomes.

The sickle red cell release of oxygenated hemoglobin into the endothelium increases the risk of oxidative stress and induces intravascular hemolysis. Furthermore, the production and release of highly reactive ferric heme and iron in the blood plasma promotes the generation of 
hydroxyl radicals and ultimately oxidative damage [27]. In a 2019 study of 147 individuals, blood analysis via biochemical testing revealed an increased level of hydroxyl radicals and nitric oxide production prevalent in the SCD group. Aberrant blood element interactions not directly affected by the $\mathrm{HbS}$ mutation paves the way for ischemic reperfusion injury and supports elevated inflammatory tone [28]. Evidence supports that alteration of hemoglobin capacity is a precursor to chronic inflammatory disease [29]. Studies that effectively target the reduction of oxidative and nitrosative stress in SCD remain in progression. Although proving the efficacy of nitric oxide inhalation therapy was unsuccessful, a new therapeutic agent that deters oxidative stress has recently been the $1^{\text {st }}$ FDA approved SCA-pathology targeting therapy since Hydroxyurea 30 years prior [14].

L-Glutamine is known for its increased uptake by sickled red cells in comparison to normal erythrocytes [30]. NAD+ uptake by cells slow oxidative damage hereby discouraging various clinical expressions. Although the pathologic mechanism is not fully understood, the efficacy of L-glutamine in reduction of VOC occurrences is an evidence-based phenomenon. In 2018, a double- blind phase 3 trial demonstrated a significant reduction in sickle cell related pain crises after receiving L-glutamine orally over the course of 48 weeks. A large portion of these participants were also noted as intolerable to hydroxyurea [31].

\section{Targeting SCA Pathophysiology}

\section{Atypical Endothelial Interactions}

Heterocellular aggregates are understood to propagate the phenomenon of vascular occlusion [32]. The lifespan of an erythrocyte in a healthy individual is 120 days and is drastically shortened to 12 days in a person with sickle cell anemia; stimulating the increased production of reticulocytes rich in adhesion molecules. Both sickled erythrocytes and reticulocytes express more endothelial adhesion relative to normal erythrocytes [33]. This phenomenon is attributed to the mechanistic role of extracellular matrix proteins and molecular interactions amongst blood formed elements and the vascular endothelium.

When leukocyte concentrations rise, so does its tendency to adhere to the endothelium, platelets and each other. Modifying the expression of adhesion on cell endothelium is currently being investigated. Clinicians have studied ways to chemically block leukocytes and other adhesion mediated molecules in order to reduce blood viscosity and impede Vaso-Occlusive pain episodes (VOC) [34]. Of note is the inhibition of the adhesion mediated molecule P-selectin, a molecule known for upregulation in endothelial cells. A placebo controlled, phase II experiment tested crizanlizumab and its effectiveness; a humanized antibody proven to bind to P-selectin and inhibit cell to cell related adhesion. A total of 198 adult patients were involved and those who received high dose crizanlizumab resulted in a significant decline $(62.9 \%)$ in sickle cell pain crisis [35]. Studies also support the benefit of an antiplatelet agent in treatment of sickle cell related complications. Prasugrel functions to inhibit platelet activation and Fernandez A | Volume 1; Issue 2 (2020) | JCMR-1(2)-010 | Review Article 
adenosine diphosphate mediated agglutination. The drug was studied in multiple phase 1 placebo controlled settings and demonstrated a reduction in platelet surface P-selectin in adults with SCA [36,37].

\section{Prevention of Erythrocyte Dehydration}

Sickled erythrocyte dehydration plays an important role in diminished organ perfusion [38]. Maintenance of intracellular volume and proper ion concentrations is imminent in avoidance of erythrocyte dehydration. Yet it is a fate in repeated sickling. Several channels are critically involved but emphasis has been placed on the irregular functioning of the Kalium-Chloride Cotransport system (KCC) and Gardos channel in SCA. KCC activity increases in proportion to repeated sickling; functioning pathologically in sickle cells to overshoot its target hemoglobin concentration and priming the reticulocyte to sickle [39]. HbS polymerization activates a nonselective cation leaky pathway in a fraction of sickle cells upon deoxygenation. Calcium permeabilization promotes membrane protein digestion via activation of proteolytic enzymes like phospholipase and protease. One clinical investigation demonstrated a notable decline in erythrocyte KCC activity and permeabilization in the presence of magnesium intercellularly. Magnesium as an oral supplement for patients with sickle cell anemia demonstrated a notable improvement in erythrocyte dehydration [40]. Nevertheless, red blood cell magnesium levels, not serum magnesium is encouraged to be used as a determinant of SCA-related hospitalizations. Evidence shows that a patient with a lower concentration of erythrocyte magnesium strongly correlates with the increased risk of more frequent VOC episodes [41].

Although pharmacologically targeting the gardos channel in-vitro has not been as successful as KCC modifications, a deepened understanding of its biochemistry has encouraged researchers to find efficient ways to upregulate potassium in SCA. Nevertheless, binding of upregulated vasoactive substance ET1, a potent vasoconstrictor, alters the mechanism of the gardos channel in an anoxic environment. Senicapoc is a gardos channel blocker that has been tested to increase intracellular potassium levels and counteract dehydration. In a phase II study, 145 patients received oral. Senicapoc over the course of 52 weeks. Data showed a notable increase in hemoglobin levels and a reduction in dense erythrocytes. Although the rate in which hemolysis improved relative to the placebo group was deemed insignificant, research is still in progress for pharmaceutically targeting erythrocyte dehydration for SCA.

\section{Stem Cell Transplantation}

Allogenic Hematopoietic Stem Cell Transplantation (HSCT) is the sole curative possibility for sickle cell patients who present with a severe clinical presentation. To date, human leukocyte antigen Matched Sibling Donor (MSD) HSCT has the highest event free survival rates of 93 to

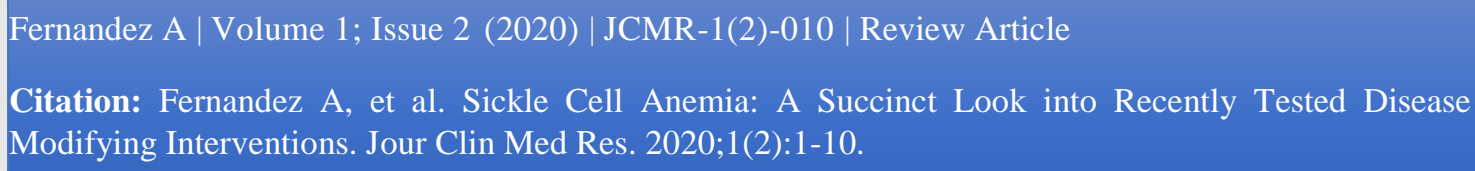


97\% [31]. Reports show that transplant recipients have absolute resolution of their sickle cell related complications; including no further organ reperfusion damage or episodes of vasoocclusion [42]. Studies even report reduced levels in toxicities from $22.6 \%$ to $3 \%$ with the use of reduced-intensity conditioning regimens such as Antithymocyte Globulin (ATG) which limit the likelihood of organ transplant rejection [35].

Notwithstanding the success of allogenic MSD-HSCT, the donor pool for engraftment recipients remain low since only $20 \%$ of minorities have a matched sibling donor [24]. The World Marrow Donor Association reports only 33\% of African Americans having a match; attributed to having a less common more diverse haplotype. The battle continues in HSCT donor pool expansion although recent efforts in using alternative donor sources has demonstrated an encouraging outcome. Of note is Matched Unrelated Donor (MUD) HSCT although mitigating occurrences of graft versus host disease remains limited with varied results. Walters et al., reported a 1 to 2 year event free survival rates of $76 \%$ and $69 \%$ respectively and overall survival of $96 \%$ and $79 \%$ in recipients of Haploidentical HSCT. Moreover, rates of Graft Versus Host Disease (GVHD) were reported to be higher (62\%) compared to having a related donor [29]. The use of post-transplant cyclophosphamide which functions to impede alloreactive T-cells in MUD HSCT has been recently used in a series of pilot studies to impede high levels of GVHD [17]. Bolanos et al., was a study that used a haploidentical sibling, parent or child donor and cyclophosphamide as a non-myeloablative conditioning regimen which resulted in a $43 \%$ graft failure rate [42]. Nevertheless, lack of larger studies on HSCT donor expansion and a standard criterion for donor recipients remain barriers treatment efficacy.

\section{Conclusion}

Although the genetic basis of sickle cell anemia is well reported, the quest for a deepened understanding of its complex pathogenesis continues. This has encouraged scientist globally to find ways in which therapeutic agents can target elements that exacerbate vaso-occlusion and hemolysis. Understanding the roles of increasing $\mathrm{HbF}$ production, prevention of cellular and sickle red cell adhesion, inflammation, no depletion and hemolysis have paved the for oral therapeutic advancements for SCD. Although there is a demand for more clinical trials and analysis, efforts toward producing effective treatment options that are available globally are at its all-time high.

\section{References}

1. Lanzkron S, Carroll CP, Haywood Jr C. The burden of emergency department use for sickle-cell disease: an analysis of the national emergency department sample database. Am J Hematol. 2010;85(10):797-9.

2. Gong L, Maiteki-Sebuguzi C, Rosenthal PJ, Hubbard AE, Drakeley CJ, Dorsey G, et al. Evidence for both innate and acquired mechanisms of protection from Plasmodium falciparum in children with sickle cell trait. Blood J Am Society Hematol. 2012;119(16):3808-14. 
3. Serjeant GR. The natural history of sickle cell disease. Cold Spring Harbor Perspect Med. 2013;3(10):a011783.

4. Manwani D, Frenette PS. Vaso-occlusion in sickle cell disease: pathophysiology and novel targeted therapies. Blood J Am Society Hematol. 2013;122(24):3892-8.

5. Hebbel RP. Beyond hemoglobin polymerization: The red blood cell membrane and sickle disease pathophysiology. Blood 1991;77(2):214-37.

6. Lew VL, Etzion Z, Bookchin RM. Dehydration response of sickle cells to sickling-induced Ca++ permeabilization. Blood J Am Society Hematol. 2002;99(7):2578-85.

7. Brousse V, Colin Y, Pereira C, Arnaud C, Odièvre MH, Boutemy A, et al. Erythroid adhesion molecules in sickle cell anaemia infants: insights into early pathophysiology. EBioMedicine. 2015;2(2):154-7.

8. Charache S, Terrin ML, Moore RD, Dover GJ, Barton FB, Eckert SV, et al. Effect of hydroxyurea on the frequency of painful crises in sickle cell anemia. New Eng J Med. 1995;332(20):1317-22.

9. Lettre G, Bauer DE. Fetal haemoglobin in sickle-cell disease: from genetic epidemiology to new therapeutic strategies. The Lancet. 2016;387(10037):2554-64.

10. Piccin A, Murphy C, Eakins E, Rondinelli MB, Daves M, Vecchiato C, et al. Insight into the complex pathophysiology of sickle cell anaemia and possible treatment. Euro J Haematol. 2019;102(4):319-30.

11. Kato GJ, Steinberg MH, Gladwin MT. Intravascular hemolysis and the pathophysiology of sickle cell disease. J Clin Invest. 2017;127(3):750-60.

12. Wang WC, Ware RE, Miller ST, Iyer RV, Casella JF, Minniti CP, et al. Hydroxycarbamide in very young children with sickle-cell anaemia: a multicentre, randomised, controlled trial (BABY HUG). The Lancet. 2011;377(9778):1663-72.

13. Diallo D, Tchernia G. Sickle cell disease in Africa. Cur Opin Hematol. 2002;9(2):111-6.

14. Opoka RO, Ndugwa CM, Latham TS, Lane A, Hume HA, Kasirye P, et al. Novel use Of Hydroxyurea in an African Region with Malaria (NOHARM): a trial for children with sickle cell anemia. Blood J Am Society Hematol. 2017;130(24):2585-93.

15. DeSimone J, Koshy M, Dorn L, Lavelle D, Bressler L, Molokie R, et al. Maintenance of elevated fetal hemoglobin levels by decitabine during dose interval treatment of sickle cell anemia. Blood J Am Society Hematol. 2002;99(11):3905-8.

16. Steinberg MH, Lu ZH, Barton FB, Terrin ML, Charache S, Dover GJ. Multicenter Study of Hydroxyurea, fetal hemoglobin in sickle cell anemia: determinants of response to hydroxyurea. Blood J Am Society Hematol. 1997;89(3):1078-88.

17. Anemia C. Adherence to hydroxyurea in children with sickle. 2014;156(3):415-19.

18. Molokie R, Lavelle D, Gowhari M, Pacini M, Krauz L, Hassan J, et al. Oral tetrahydrouridine and decitabine for non-cytotoxic epigenetic gene regulation in sickle cell disease: a randomized phase 1 study. PLoS Med. 2017;14(9):1-28.

19. Rees DC, Williams TN, Gladwin MT. Sickle-cell disease. The Lancet. 2010;376(9757):2018-31.

20. Piccin A, Murphy C, Eakins E, Rondinelli MB, Daves M, Vecchiato C, et al. Insight into the complex pathophysiology of sickle cell anaemia and possible treatment. Euro J Haematol. 2019;102(4):319-30.

Fernandez A | Volume 1; Issue 2 (2020) | JCMR-1(2)-010 | Review Article

Citation: Fernandez A, et al. Sickle Cell Anemia: A Succinct Look into Recently Tested Disease Modifying Interventions. Jour Clin Med Res. 2020;1(2):1-10. 
21. Kato GJ, Steinberg MH, Gladwin MT. Intravascular hemolysis and the pathophysiology of sickle cell disease. J Clin Invest. 2017;127(3):750-60.

22. Haque AK, Gokhale S, Rampy BA, Adegboyega P, Duarte A, Saldana MJ. Pulmonary hypertension in sickle cell hemoglobinopathy: a clinicopathologic study of 20 cases. Human Pathol. 2002;33(10):1037-43.

23. Voskou S, Aslan M, Fanis P, Phylactides M, Kleanthous M. Oxidative stress in $\beta$-thalassaemia and sickle cell disease. Redox Biol. 2015;6:226-39.

24. Biswal S, Rizwan H, Pal S, Sabnam S, Parida P, Pal A. Oxidative stress, antioxidant capacity, biomolecule damage, and inflammation symptoms of sickle cell disease in children. Hematol. 2019;24(1):1-9.

25. Niihara Y, Zerez CR, Akiyama DS, Tanaka KR. Oral L-glutamine therapy for sickle cell anemia: I. Subjective clinical improvement and favorable change in red cell NAD redox potential. Am J Hematol. 1998;58(2):117-21.

26. Niihara Y, Miller ST, Kanter J, Lanzkron S, Smith WR, Hsu LL, et al. A phase 3 trial of l-glutamine in sickle cell disease. New Eng J Med. 2018;379(3):226-35.

27. Kaul DK, Finnegan E, Barabino GA. Sickle red cell-endothelium interactions. Microcirculation. 2009;16(1):97-111.

28. Sandor B, Marin M, Lapoumeroulie C, Rabaï M, Lefevre SD, Lemonne N, et al. Effects of Poloxamer 188 on red blood cell membrane properties in sickle cell anaemia. Brit J Haematol. 2016;173(1):145-9.

29. Ataga KI, Kutlar A, Kanter J, Liles D, Cancado R, Friedrisch J, et al. Crizanlizumab for the prevention of pain crises in sickle cell disease. New Eng J Med. 2017;376(5):429-39.

30. Jakubowski JA, Zhou C, Small DS, Winters KJ, Lachno DR, Frelinger III AL, et al. A phase 1 study of prasugrel in patients with sickle cell disease: pharmacokinetics and effects on ex vivo platelet reactivity. British J Clin Pharmacol. 2013;75(6):1433-44.

31. Wun T, Soulieres D, Frelinger AL, Krishnamurti L, Novelli EM, Kutlar A, et al. A double-blind, randomized, multicenter phase 2 study of prasugrel versus placebo in adult patients with sickle cell disease. J Hematol Oncol. 2013;6(1):17.

32. Ballas SK, Lieff S, Benjamin LJ, Dampier CD, Heeney MM, Hoppe C, et al. Definitions of the phenotypic manifestations of sickle cell disease. Am J Hematol. 2010;85(1):6-13.

33. Lew VL, Etzion Z, Bookchin RM. Dehydration response of sickle cells to sickling-induced Ca++ permeabilization. Blood J Am Society Hematol. 2002;99(7):2578-85.

34. De Franceschi L, Bachir D, Galacteros F, Tchernia G, Cynober T, Alper S, et al. Oral magnesium supplements reduce erythrocyte dehydration in patients with sickle cell disease. J Clinl Invest. 1997;100(7):1847-52.

35. Yousif OO, Al-Naama LM. Red blood cell and serum magnesium levels among children and adolescents with sickle cell anemia. Biological Trace Elem Res. 2018;186(2):295-304.

36. Brugnara C. Sickle cell dehydration: Pathophysiology and therapeutic applications. Clin Hemorheol Microcircul. 2018;68(2-3):187-204.

37. Gluckman E. Allogeneic transplantation strategies including haploidentical transplantation in sickle cell disease. Hematology. Am Society Hematol/Education Program Book. 2013;2013(1):370-6.

38. Roth M, Krystal J, Manwani D, Driscoll C, Ricafort R. Stem cell transplant for children with sickle cell anemia: parent and patient interest. Biol Blood Marrow Transplant. 2012;18(11):1709-15. 
39. Bernaudin F, Socie G, Kuentz M, Chevret S, Duval M, Bertrand Y, et al. Long-term results of related myeloablative stem-cell transplantation to cure sickle cell disease. Blood $\mathrm{J}$ Am Society Hematol. 2007;110(7):2749-56.

40. Horan JT, Liesveld JL, Fenton P, Blumberg N, Walters MC. Hematopoietic stem cell transplantation for multiply transfused patients with sickle cell disease and thalassemia after low-dose total body irradiation, fludarabine, and rabbit anti-thymocyte globulin. Bone Marrow Transplant. 2005;35(2):171-7.

41. Adamkiewicz TV, Mehta PS, Boyer MW, Kedar A, Olson TA, Olson E, et al. Transplantation of unrelated placental blood cells in children with high-risk sickle cell disease. Bone Marrow Transplant. 2004;34(5):405-11.

42. Bolaños-Meade J, Fuchs EJ, Luznik L, Lanzkron SM, Gamper CJ, Jones RJ, et al. HLA-haploidentical bone marrow transplantation with posttransplant cyclophosphamide expands the donor pool for patients with sickle cell disease. Blood J Am Society Hematol. 2012;120(22):4285-91. 